\title{
Striped Parameterized Tube Model Predictive Control
}

\author{
Diego Muñoz-Carpintero ${ }^{\mathrm{a}, 1}$ Basil Kouvaritakis ${ }^{\mathrm{b}}$ Mark Cannon ${ }^{\mathrm{b}}$
}

${ }^{a}$ School of Electrical and Electronic Engineering, Nanyang Technological University, 639798, Singapore

${ }^{\mathrm{b}}$ Department of Engineering Science, University of Oxford, Oxford OX1 3PJ, UK

\begin{abstract}
A modification of the Parameterized Tube Model Predictive Control (PTMPC) strategy for linear systems with additive disturbances is proposed, which reduces the dependence of the number of optimization variables on horizon length from quadratic to linear by using a triangular striped prediction structure. Unlike PTMPC, which assumes a fixed linear terminal feedback law for predictions, the proposed prediction scheme allows disturbance compensation to extend beyond the initial $N$-step prediction horizon. The resulting scheme can potentially outperform PTMPC in terms of the size of the domain of attraction and allows for a longer horizon $N$ for the same computational demand.
\end{abstract}

Keywords: Predictive control; Uncertain linear systems; Bounded disturbances; Robust Control; Constraints.

\section{Introduction}

Robust model predictive control (RMPC) of linear systems subject to additive uncertainty is an imporant area of research. Optimal RMPC requires the solution of dynamic programming [1] or minimax problems [12] and is computationally intractable, thus a compromise between sub-optimality and complexity is necessary. Early RMPC considered open-loop solutions, where a single input sequence is determined for all disturbance realizations. This is conservative because it ignores information on future states and uncertainties that will be available for use by the controller and can thus lead to poor performance and infeasibility. Quasi-closed loop formulations on the other hand optimize perturbations on a pre-stabilizing law [6], [4]. They provide an improvement, but are still conservative because pre-stabilization is designed offline.

Optimality is improved upon by affine-in-the-disturbance MPC (ADMPC) [7], [2]. This uses feedforward plus linear disturbance compensation with a triangular structure in the near horizon, while in the far horizon it deploys fixed state feedback. ADMPC has been superseded by parameterized tube MPC (PTMPC) [11] which exploits a separable triangular prediction structure of partial tubes, the first of which describes the nominal dynamics and the rest are associated with future disturbances. These are unknown and are defined in terms of the vertices of the allowable set of disturbances. The PTMPC policy is piecewise-affine-in-the-disturbance and hence leads to larger domains of attraction than ADMPC. The number of variables and constraints grows quadratically with the prediction horizon $N$ in both ADMPC and PTMPC which therefore are limited to low-dimensional systems or small $N$.

Here we present a RMPC formulation that is a modification of PTMPC in which the degrees of freedom affect (directly) the inputs over the entire prediction horizon with a striped structure. This idea has been explored before but in the context of constructing parameterized robust control invariant sets through the use of a sequence contracting technique [9]. It has also been explored in Stochastic MPC [3], where affinein-the-disturbance compensation extends over the infinite far prediction horizon according to a fixed control law computed offline. In our approach the disturbance compensation in the far horizon is computed online, and leads to a terminal con-

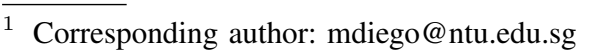

trol law more general than linear feedback. This is achieved by expressing predictions as the sum of a nominal sequence and a single sequence associated with all particular future disturbances. This gives rise to a separable striped disturbance compensation scheme which is allowed to extend over an infinite prediction horizon and leads to a number of variables and constraints which grows linearly with $N$ (rather than quadratically as for PTMPC). Additionally, allowing for disturbance compensation into the far horizon implies a constraint relaxation. Simulations show that this strategy can, for a comparable number of degrees of freedom and constraints, lead to larger domains of attraction.

Section 2 gives the system description and a brief review of the separable scheme of [11]. Our strategy is introduced in Section 3 , and Section 4 analyses the control theoretic properties of two alternative strategies: one with exponential convergence to a known minimal robust invariant set; and another based on input-to-state stability, as presented in [8], which enjoys increased control authority but does not have the guarantee of convergence to the minimal robust invariant set. Section 5 presents an illustration by simulation of the benefits of the proposed strategy and conclusions are drawn in Section 6.

Notation: $\mathbb{N}_{+}$and $\mathbb{R}_{+}$denote the sets of positive integers and positive reals. $\mathbb{N}=\mathbb{N}_{+} \cup\{0\}$ and $\mathbb{N}_{[a, b]}=\{a, a+$ $1, \ldots, b\}$. For $X, Y \subset \mathbb{R}^{n}, X \oplus Y=\{x+y: x \in X, y \in$ $Y\}$ denotes the Minkowski sum, and the image of $X$ un$\operatorname{der} M \in \mathbb{R}^{m \times n}$ is $M X=\{M x: x \in X\}$. For $X=$ $\operatorname{conv}\left(\left\{x_{1}, \ldots, x_{n}\right\}\right)($ where $\operatorname{conv}(\cdot)$ denotes the convex hull) and $A, B \in \mathbb{R}^{m \times n},(A, B) X=\operatorname{conv}(\{(A x, B x): x \in X\})$. For $Y=\left\{y: E_{l} y \leq 1, l \in \mathbb{N}_{\left[1, n_{y}\right]}\right\}$, the $Y$-distance function of $x$ is $\operatorname{dist}_{Y}(x)=\max \left(\left\{E_{l} x-1: l \in \mathbb{N}_{\left[1, n_{y}\right]}\right\} \cup\{0\}\right)$, and the maximum $Y$-distance function of $X$ is $\operatorname{maxdist}_{Y}(X)=$ $\max \left(\left\{E_{l} x-1: x \in X, l \in \mathbb{N}_{\left[1, n_{y}\right]}\right\} \cup\{0\}\right)$.

\section{System description and Separable Prediction Scheme}

Consider the linear, discrete-time system and constraints

$$
\begin{aligned}
& x^{+}=A x+B u+w, \\
& F x+G u \leq 1,
\end{aligned}
$$

with $x \in \mathbb{R}^{n_{x}}, u \in \mathbb{R}^{n_{u}}, w \in \mathbb{W} \subset \mathbb{R}^{n_{x}} . F \in \mathbb{R}^{n_{c} \times n_{x}}$ and $G \in \mathbb{R}^{n_{c} \times n_{u}}$. Here $\mathbb{W}=\operatorname{conv}\left(\left\{\tilde{w}_{i}: i \in \mathbb{N}_{[1, q]}\right\}\right)$ is a polytope that contains the origin and $\mathbb{Y}=\{y=(x, u)$ : $\left.F_{l} x+G_{l} u \leq 1, l \in \mathbb{N}_{\left[1, n_{c}\right]}\right\}$ is a polytope that contains the 
origin in its interior; the subscript $l$ denotes the $l^{\text {th }}$ row.

A robust MPC strategy with a separable prediction scheme is presented in [11] in which, since the system is linear, the predictions can be split in sequences, one associated with the nominal dynamics $(w \equiv 0)$ and the rest associated with each future disturbance. The $0^{\text {th }}$ partial state and control sequences $\mathbf{x}_{(0,:)}=\left\{x_{(0, k)}\right\}_{k \in \mathbb{N}}$ and $\mathbf{u}_{(0,:)}=\left\{u_{(0, k)}\right\}_{k \in \mathbb{N}}$ account for the nominal dynamics satisfying at each prediction time $k \in \mathbb{N}$

$$
x_{(0, k+1)}=A x_{(0, k)}+B u_{(0, k)} \text {, with } x_{(0,0)}=x \text {, }
$$

where $x$ is the current state. Similarly, the dynamical contribution of the disturbance acting at the $(j-1)^{\text {th }}$ prediction time, $w_{j-1}, j \in \mathbb{N}_{+}$is given by the $j^{\text {th }}$ partial state and control sequences, $\mathbf{x}_{(j,:)}=\left\{x_{(j, k)}\right\}_{k \in \mathbb{N}_{[j, \infty)}}$ and $\mathbf{u}_{(j,:)}=$ $\left\{u_{(j, k)}\right\}_{k \in \mathbb{N}_{[j, \infty)}}$, which for $k \in \mathbb{N}_{j, \infty}$ satisfy

$$
x_{(j, k+1)}=A x_{(j, k)}+B u_{(j, k)} \text {, with } x_{(j, j)}=w_{j-1} \text {. }
$$

Since $w_{j-1}$ is unknown, the predictions of $x_{(j, k)}$ and $u_{(j, k)}$ are also unknown, however it is possible to find exactly the sets that contain them. Considering the extreme realizations, $\tilde{w}_{i}, i \in \mathbb{N}_{[1, q]}$, of $w_{j-1}$, and denoting corresponding $j^{\text {th }}$ partial extreme state and control sequences as $\mathbf{x}_{(i, j,:)}=\left\{x_{(i, j, k)}\right\}_{k \in \mathbb{N}_{[j, \infty)}}$ and $\mathbf{u}_{(i, j,:)}=\left\{u_{(i, j, k)}\right\}_{k \in \mathbb{N}_{[j, \infty)}}$, we define $u_{(j, k)}=\sum_{i=1}^{q} \lambda_{i}\left(w_{j-1}\right) u_{(i, j, k)}$ in terms of convex interpolation parameters $\lambda_{i}\left(w_{j-1}\right), i \in \mathbb{N}_{[1, q]}$ such that $w_{j-1}=\sum_{i=1}^{q} \lambda_{i}\left(w_{j-1}\right) \tilde{w}_{i}$, and it follows that

$$
\begin{aligned}
& x_{(j, k)} \in X_{(j, k)}=\operatorname{conv}\left(\left\{x_{(i, j, k)}: i \in \mathbb{N}_{[1, q]}\right\}\right), \\
& u_{(j, k)} \in U_{(j, k)}=\operatorname{conv}\left(\left\{u_{(i, j, k)}: i \in \mathbb{N}_{[1, q]}\right\}\right),
\end{aligned}
$$

where the dynamics of the $j^{\text {th }}$ partial extreme state and control sequences for $i \in \mathbb{N}_{[1, q]}, j \in \mathbb{N}_{+}$, are given by

$$
\begin{aligned}
& x_{(i, j, j)}=\tilde{w}_{i}, \\
& x_{(i, j, k+1)}=A x_{(i, j, k)}+B u_{(i, j, k)}, \quad k \in \mathbb{N}_{[j, \infty)} .
\end{aligned}
$$

Then the full predictions are given by

$$
x_{k}=\sum_{j=0}^{k} x_{(j, k)}, \quad u_{k}=\sum_{j=0}^{k} u_{(j, k)}, \quad \forall k \in \mathbb{N}
$$

thus defining a triangular prediction structure as shown for the inputs in Figure 1 (the case for the states is analog), where from (5) and (7), the full predicted inputs are contained in sets given by the Minkowski sum of the elements in each column.

Assumption 1 (i) The pair $(A, B)$ is stabilizable; ii) The matrix gain $K$ is such that $\Phi=A+B K$ is strictly stable and the minimal robust invariant set of (1) under $u=K x, \Omega_{K}^{\infty}$, satisfies $(I, K) \Omega_{K}^{\infty} \in$ interior $(\mathbb{Y})$.

A control policy is defined implicitly in this separable prediction scheme. From (7) the predicted inputs can be re-written as $u_{k}=u_{(0, k)}+\sum_{j=1}^{k} u_{(j, k)}$, where $u_{(j, k)}$ is a convex interpolation of $u_{(i, j, k)}, i \in \mathbb{N}_{[1, q]}$ depending on the value of $w_{(j-1)}$. Thus, the associated control policy is piecewise-affine-in-thedisturbance [11]. Note that there is no need to know the interpolation parameters, as we only need to know the extreme trajectories to guarantee feasibility.

\begin{tabular}{lllll|ll} 
Control tubes & & & Mode 1 & Mode 2 & \\
$u_{(0,0)}$ & $u_{(0,1)}$ & $u_{(0,2)}$ & $\cdots$ & $u_{(0, N-1)}$ & $K x_{(0, N)}$ & $\cdots$ \\
& $U_{(1,1)}$ & $U_{(1,2)}$ & $\cdots$ & $U_{(1, N-1)}$ & $K X_{(1, N)}$ & $\cdots$ \\
& & $U_{(2,2)}$ & $\cdots$ & $U_{(2, N-1)}$ & $K X_{(2, N)}$ & $\cdots$ \\
& & & & $\vdots$ & \\
& & & & $U_{(N-1, N-1)}$ & $K X_{(N-1, N)}$ & $\cdots$ \\
& & & & & $K X_{(N, N)}$ & $\ddots$ \\
\hline$u_{0}$ & $u_{1}$ & $u_{2}$ & $\cdots$ & $u_{N-1}$ & $u_{N}$ &
\end{tabular}

Fig. 1. Triangular prediction scheme of PTMPC - Inputs

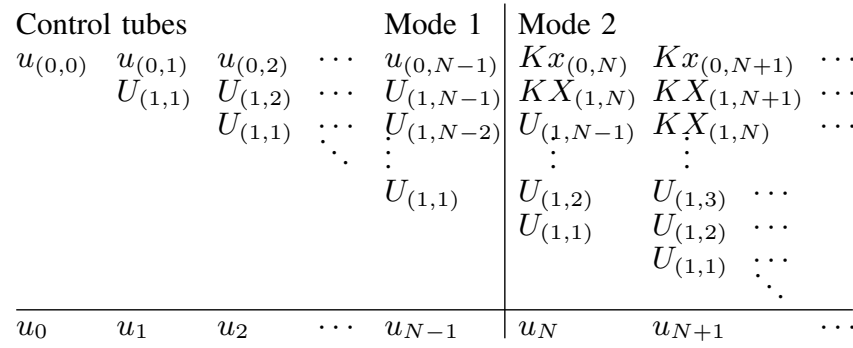

Fig. 2. Triangular prediction scheme of Striped PTMPC - Inputs

The first $N$ prediction steps are referred to as Mode 1 and the remainder as Mode 2, where it is usual to deploy a stabilizing terminal control law. Then, in [11], $x_{(0, k)}, u_{(0, k)}, x_{(i, j, k)}$ and $u_{(i, j, k)}$ are free optimization variables in Mode 1 , while in Mode $2 u_{(0, k)}$ and $u_{(i, j, k)}$ are associated to the terminal control law $u=K x$ and are given by $u_{(0, k)}=K x_{(0, k)}$ and $u_{(i, j, k)}=$ $K x_{(i, j, k)}$ (see Figure 1). On the other hand, a striped structure to reduce the number of variables and constraints is invoked in this paper (see Figure 2). The details are presented next.

\section{The RMPC Strategy: Striped prediction scheme and constraints}

Consider the following structural constraints in addition to (3)(7): for $j \in \mathbb{N}_{+}, k \in \mathbb{N}_{[j, \infty)}, i \in \mathbb{N}_{[1, q]}$

$$
u_{(i, j, k)}:=u_{(i, 1, k-j+1)}, \quad x_{(i, j, k)}=x_{(i, 1, k-j+1)},
$$

which imply that $X_{(j, k)}=X_{(1, k-j+1)}$ and $U_{(j, k)}=$ $U_{(1, k-j+1)}$, so that the elements on each diagonal below the first row (see Figure 2) are the same, yielding a striped structure. The $1^{\text {st }}$ partial extreme state and controls $x_{(i, 1, k)}, u_{(i, 1, k)}$ are free optimization variables for $k \in \mathbb{N}_{[1, N]}$ and $k \in \mathbb{N}_{[1, N-1]}$, respectively, and we fix $u_{(i, 1, k)}=K x_{(i, 1, k)}$ for $k \in \mathbb{N}_{[N, \infty)}$. Thus all the $j^{\text {th }}$ partial sequences are fully defined by the $1^{\text {st }}$ partial extreme sequences $\mathbf{x}_{(i, 1, N)}=\left\{x_{(i, 1, k)}\right\}_{k \in \mathbb{N}_{[1, N]}}$ and $\mathbf{u}_{(i, 1, N-1)}=\left\{u_{(i, 1, k)}\right\}_{k \in \mathbb{N}_{[1, N-1]}}$. The $k$-step-ahead predictions are bounded by sets given by the Minkowski sum of the nominal $k$-step-ahead predictions and all the $X_{(1, j)}$ or $U_{(1, j)}$ for $j \in \mathbb{N}_{[1, k]}$. Then, $\forall k \in \mathbb{N}$

$$
x_{k} \in x_{(0, k)} \oplus \bigoplus_{j=1}^{k} X_{(1, j)}, u_{k} \in u_{(0, k)} \oplus \bigoplus_{j=1}^{k} U_{(1, j)},
$$

and the relevant prediction dynamics are described by

$$
\begin{array}{ll}
x_{(0,0)}=x, & \\
x_{(0, k+1)}=A x_{(0, k)}+B u_{(0, k)}, & k \in \mathbb{N}_{[0, N-1]}, \\
x_{(0, k+1)}=\Phi x_{(0, k)}, & k \in \mathbb{N}_{[N, \infty)}, \\
x_{(i, 1,1)}=\tilde{w}_{i}, & \\
x_{(i, 1, k+1)}=A x_{(i, 1, k)}+B u_{(i, 1, k)}, & k \in \mathbb{N}_{[1, N-1]} \\
x_{(i, 1, k+1)}=\Phi x_{(i, 1, k)}, & k \in \mathbb{N}_{[N, \infty)} .
\end{array}
$$

Remark 2 From (8) and (9) the predicted inputs $u_{k}, k \in \mathbb{N}_{+}$ depend on the $1^{\text {st }}$ partial extreme sequences $\mathbf{u}_{(i, 1, N-1)}$ which are degrees of freedom. This implies that the disturbance compensation extends to Mode 2 throughout the infinite horizon, and thus the terminal control law is not fixed. This setting is different than just reducing the number of decision variables by forcing a striped structured on the parameterization of [11], since it only allows compensation for $k \in \mathbb{N}_{[0, N-1]}$.

The separable scheme of (8)-(10) defines the overall mixed state and control tubes $\mathrm{Y}=\left\{Y_{k}\right\}_{k \in \mathbb{N}}$

$$
Y_{k}=y_{(0, k)} \oplus Y_{(1,1)} \oplus \ldots \oplus Y_{(1, k)},
$$


where the mixed state/control $y_{(0, k)}$ and $Y_{(1, j)}$ are

$$
\begin{array}{ll}
y_{(0, k)}=\left\{\left(x_{(0, k)}, u_{(0, k)}\right)\right\} & k \in \mathbb{N}_{[0, N-1]}, \\
y_{(0, k)}=\left\{\left(x_{(0, k)}, K x_{(0, k)}\right)\right\}, & k \in \mathbb{N}_{[N, \infty)}, \\
Y_{(1, j)}=\operatorname{conv}\left(\left\{\left(x_{(i, 1, j)}, u_{(i, 1, j)}\right): i \in \mathbb{N}_{[1, q]}\right\}\right),
\end{array}
$$

for $j \in \mathbb{N}_{[1, N-1]}$, and

$$
Y_{(1, j)}=\operatorname{conv}\left(\left\{\left(x_{(i, 1, j)}, K x_{(i, 1, j)}\right): i \in \mathbb{N}_{[1, q]}\right\}\right),
$$

for $j \in \mathbb{N}_{[N, \infty)}$. Eq. (11) can be rewritten in terms of $\left\{y_{(0, k)}\right\}_{k \in \mathbb{N}_{[0, N-1]}}$ and $\left\{Y_{(1, j)}\right\}_{j \in \mathbb{N}_{[1, N-1]}}$ alone, which depend on the optimization variables $\mathbf{x}_{(i, 1, N)}$ and $\mathbf{u}_{(i, 1, N-1)}$ :

$$
\begin{aligned}
& Y_{k}=y_{(0, k)} \oplus \bigoplus_{j=1}^{k} Y_{(1, j)}, \quad k \in \mathbb{N}_{[0, N-1]}, \\
& Y_{k}=(I, K) \Phi^{k-N} x_{(0, N)} \oplus \bigoplus_{j=1}^{N-1} Y_{(1, j)} \oplus \\
& \bigoplus_{j=N}^{k}(I, K) \Phi^{j-N} X_{(1, N)}, \quad k \in \mathbb{N}_{[N, \infty)},
\end{aligned}
$$

where $X_{(1, N)}=\operatorname{conv}\left(\left\{x_{(i, 1, N)}: i=1, . ., q\right\}\right)$, and $I$ is the identity; note that $Y_{(1, j)}=(I, K) X_{(1, j)}$ for $j \in \mathbb{N}_{[N, \infty)}$.

The condition for constraint satisfaction is that

$$
Y_{k} \subseteq \mathbb{Y}, \quad \forall k \in \mathbb{N}
$$

which implies an infinite number of constraints. Instead, constraints will be enforced explicitly for $k \in \mathbb{N}_{\left[0, N+N_{2}-1\right]}$, whereas for $k \in \mathbb{N}_{\left[N+N_{2}, \infty\right)}$ use will be made of a terminal constraint on the nominal and uncertain sequences at the $\left(N+N_{2}\right)^{\text {th }}$ prediction instant.

Proposition 3 Let $\Omega_{0}$ be an invariant set for the nominal $d y$ namics of (10c) and let $\Omega_{1}$ be an arbitrary polytopic set that contains the origin. Then the conditions

$$
\begin{aligned}
x_{\left(0, N+N_{2}\right)} & \in \Omega_{0}, \\
X_{\left(1, N+N_{2}\right)} & \subseteq \Omega_{1},
\end{aligned}
$$

imply that $Y_{k} \subseteq \bar{Y}_{\infty}$ for all $k \in \mathbb{N}_{\left[N+N_{2}, \infty\right)}$, where

$$
\bar{Y}_{\infty}=(I, K) \Omega_{0} \oplus \bigoplus_{j=1}^{N-1} Y_{(1, j)} \oplus \bigoplus_{j=N}^{N+N_{2}-1}(I, K) \Phi^{j-N} X_{(1, N)}
$$

and $\Omega_{1}^{\infty}$ is the minimal invariant set for the system with $d y$ namics $z^{+}=\Phi z+w$, with $w \in \Omega_{1}$.

Proof: This follows from: (i) $y_{(0, k)}=\left\{\left(x_{(0, k)}, K x_{(0, k)}\right)\right\}$ $\subseteq(I, K) \Omega_{0}, k \in \mathbb{N}_{\left[N+N_{2}, \infty\right)}$ for $x_{\left(0, N+N_{2}\right)} \in \Omega_{0}$ with $\Omega_{0}$ invariant for (10c); and (ii) $\oplus_{j=N+N_{2}}^{k} \Phi^{k-\left(N+N_{2}\right)} X_{\left(1, N+N_{2}\right)}$ $\subseteq \Omega_{1}^{\infty}$, so that $\oplus_{j=N+N_{2}}^{k}(I, K) \Phi^{k-\left(N+N_{2}\right)} X_{\left(1, N+N_{2}\right)} \subseteq$ $(I, K) \Omega_{1}^{\infty}$.

The knowledge of a set that contains the state and input predictions $\forall k \in \mathbb{N}_{\left[N+N_{2}, \infty\right)}$, enables the use of a finite number of constraints to ensure constraint satisfaction:

Corollary 4 Under the conditions of Proposition 3, satisfaction of (16),

$$
\begin{aligned}
& Y_{k} \subseteq \mathbb{Y}, \quad k \in \mathbb{N}_{\left[0, N+N_{2}-1\right]}, \text { and } \\
& \bar{Y}_{\infty} \subseteq \mathbb{Y},
\end{aligned}
$$

imply the satisfaction of (15).

Assumption $5 \Omega_{1}=\Phi^{N+N_{2}-1} \mathbb{W}$ and $\Omega_{0}$ is a robust positively invariant set for $z^{+}=\Phi z+w, w \in \Phi \Omega_{1}$.
Remark 6 Assumption 5 satisfies the conditions of Proposition 3. Also, the choice of $\Omega_{1}$ guarantees that (16b) is satisfied when the partial sequences are given by $u_{(i, 1, j)}=K x_{(i, 1, j)}$, and the condition on $\Omega_{0}$ guarantees recursive feasibility (as will be discussed in the proof of Theorem 11). Note that $\Omega_{0}$ should be chosen to be small in order to relax (18), but not too small because in that case a large $N_{2}$ would be required to satisfy (16a). A sensible choice for $\Omega_{0}$, in terms of the size of the resulting domain of attraction, is given by

$$
\Omega_{0}=\alpha \bar{\Omega}_{K} \oplus \tilde{\Omega}_{\Phi \Omega_{1}}^{\infty},
$$

where $0<\alpha \leq 1$ is a scalar, $\bar{\Omega}_{K}$ is the maximal invariant set for $x^{+}=\Phi x$ s.t. (2) under $u=K x$, and $\tilde{\Omega}_{\Phi \Omega_{1}}^{\infty}$ is an invariant approximation of the minimal robust invariant set [10] for $z^{+}=\Phi z+w, w \in \Phi \Omega_{1}$. Then $\Omega_{0}$ is clearly robustly invariant for $z^{+}=\Phi z+w, w \in \Phi \Omega_{1}$. The motivation for this construction is as follows. In the absence of disturbances $\Omega_{0}$ would have to be chosen to be $\bar{\Omega}_{K}$ so as to relax (16a). However, since (16) is applied at $k=N+N_{2}, \Omega_{0}$ might be too large thereby causing (18) to be too tight. To avoid this we introduce the scaling factor $\alpha$ whose size should be commensurate with the contraction provided by $\Phi^{N_{2}}$. In the presence of disturbances however, recursive feasibility requires that $\Omega_{0}$ accounts for these disturbances, justifying the inclusion of the second term in the RHS of (19). This term has to be made to be as small as possible in order to relax (18), hence it is taken to be the minimal robust invariant set. Since the minimal robust invariant set however may not have a finite description it is replaced in (19) by a robustly invariant approximation $\tilde{\Omega}_{\Phi \Omega_{1}}^{\infty}$. Similarly, to reduce computational complexity $\bar{\Omega}_{K}$ can be replaced by an invariant approximation.

\section{The RMPC Strategy and its properties}

The stability notions of [11] are not valid here because the terminal control law is not fixed. Therefore, we examine two variations of the strategy that yield different stability properties. The first one provides convergence to a terminal invariant set for $u=K x$, which is relevant in case $u=K x$ is optimal in some sense, but it uses a constraint that may incur some conservativeness. The second variation does not impose this constraint and enables the assertion of input-to-state stability.

\subsection{Distance based cost and exponential convergence to min- imal invariant set for $u=K x$}

Let $\theta$ be the vector of all the variables in our RMPC formulation, i.e. $\mathbf{x}_{(0, N)}, \mathbf{u}_{(0, N-1)}, \mathbf{x}_{(i, 1, N)}$ and $\mathbf{u}_{(i, 1, N-1)}, i \in \mathbb{N}_{[1, q]}$. Consider the objective function

$$
J(\theta)=\sum_{k=0}^{N+N_{2}-1} \operatorname{maxdist}_{X_{f}}\left(X_{k}\right)
$$

where $X_{f}=\left\{x: H_{l}^{f} x \leq 1, l \in \mathbb{N}_{\left[1, n_{f}\right]}\right\}$ is a robustly invariant set for system (1) subject to (2) under $u=K x$. Components of the type maxdist $K X_{f}\left(U_{k}\right)$ can also be included in (20) to penalize control activity. However, this inclusion is omitted for simplicity. Also, impose that all the predicted states from $N+N_{2}$ to $\infty$ are inside $X_{f}$. Since $\operatorname{Proj}_{x}\left(\bar{Y}_{\infty}\right)$ contains all predicted states from $N+N_{2}$ to $\infty$, this can be cast as

$$
\operatorname{Proj}_{x}\left(\bar{Y}_{\infty}\right) \subseteq X_{f}
$$

Remark 7 The choice $X_{f}=\Omega_{K}$, where $\Omega_{K}$ is the maximal robustly invariant set for system (1) s.t. (2) under $u=K x$, is convenient for relaxing (21). On the other hand, invariant sets defined by few inequalities, $n_{f}$, are computationally convenient in view of (21). Thus a suitable compromise between the size of $X_{f}$ and $n_{f}$ should be chosen. 
The admissible parameter set is given by $\Theta(x)=\{\theta$ : s.t. (10a),(10b),(10d),(10e),(16),(17),(18),(21) hold\}, and the domain of attraction is given by $\mathcal{X}=\{x: \Theta(x) \neq \emptyset\}$.

Assumption $8 N, N_{2}, \Omega_{0}, X_{f}$ are such that $\Omega_{0} \oplus \Omega_{K}^{\infty} \subseteq X_{f}$.

Remark 9 This assumption can always be satisfied provided that Assumption 1 is satisfied. From Assumption 1 it follows that there exists a robustly invariant set $X_{f}$ for system (1) s.t. (2) under $u=K x$, such that $\Omega_{K}^{\infty} \subseteq \operatorname{interior}\left(X_{f}\right)$. Then $\bar{N}$ must exist such that for all $N+N_{2} \geq \bar{N}, \Omega_{1}=\Phi^{N+N_{2}-1} \mathbb{W}$ is small enough to ensure that $\Omega_{0}$ can be chosen to be small enough such that $(I, K) \Omega_{0} \oplus \Omega_{K}^{\infty} \subseteq(I, K) X_{f} \subseteq \mathbb{Y}$. If all the predicted inputs are fixed according to $u=K x$, then $\bar{Y}_{\infty}=(I, K) \Omega_{0} \oplus \bigoplus_{j=0}^{\infty}(I, K) \Phi^{j} \mathbb{W}=(I, K)\left(\Omega_{0} \oplus \Omega_{K}^{\infty}\right) \subseteq$ $\mathbb{Y}$. This guarantees that for any $x \in X_{f}$, if $\Omega_{0} \oplus \Omega_{K}^{\infty} \subseteq X_{f}$, the solution generated by setting $u=K x$ over the entire prediction, i.e. $u_{(0, k)}=K x_{(0, k)}$ and $u_{(i, 1, k)}=K x_{(i, 1, k)} \forall k \in$ $\mathbb{N}_{[1, N-1]}$ and $i \in \mathbb{N}_{[1, q]}$, will be feasible, which guarantees that $X_{f} \subseteq \mathcal{X} . X_{f}$ contains the origin in its interior since it is an invariant set for a contractive system, which implies that $\mathcal{X}$ contains the origin in its interior.

The optimal control problem $\mathcal{P}(x)$ is defined by

$$
\begin{aligned}
& V^{*}(x)=\min _{\theta} J(\theta), \text { s.t. } \quad \theta \in \Theta(x) . \\
& \theta^{*}(x)=\arg \min _{\theta} J(\theta), \text { s.t. } \quad \theta \in \Theta(x) .
\end{aligned}
$$

where $V^{*}(x)$ is the value function and $\theta^{*}(x)$ is the optimal arguments function. The optimal $\theta^{*}(x)$ is not necessarily unique because $\mathcal{P}(x)$ is a linear program as will be seen below, thus the control law is defined as

$$
\kappa^{0}(x)=u_{(0,0)}^{0}(x) \text {, }
$$

where $u_{(0,0)}^{0}(x)$ is a unique selection of $u_{(0,0)}^{*}(x)$ (which is part of the optimal $\theta^{*}(x)$ ). The control input is then set to $u:=\kappa^{0}(x)$.

In order to implement $\mathcal{P}(x)$ note that the inclusion conditions (16)-(18) and (21) can be written as linear constraints by using slack variables [11] that account for the worst case extreme partial state and control sequences. Thus (17) can be invoked equivalently as follows: for $l \in \mathbb{N}_{\left[1, n_{c}\right]}$ and $k \in \mathbb{N}_{[0, N-1]}$,

$$
\begin{aligned}
& F_{l} x_{(0, k)}+G_{l} u_{(0, k)}+\sum_{j=1}^{k} f_{(l, 1, j)} \leq 1 \\
& f_{(l, 1, j)} \geq F_{l} x_{(i, 1, j)}+G_{l} u_{(i, 1, j)}, \quad i \in \mathbb{N}_{[1, q]}, j \in \mathbb{N}_{[1, N-1]}
\end{aligned}
$$

and for $l \in \mathbb{N}_{\left[1, n_{c}\right]}$ and $k \in \mathbb{N}_{\left[N, N+N_{2}-1\right]}$,

$$
\begin{array}{r}
\left(F_{l}+G_{l} K\right) \Phi^{k-N} x_{(0, N)}+\sum_{j=1}^{k} f_{(l, 1, j)} \leq 1, \\
f_{(l, 1, j)} \geq\left(F_{l}+G_{l} K\right) \Phi^{j-N} x_{(i, 1, N)}, \quad i \in \mathbb{N}_{[1, q]}, \\
j \in \mathbb{N}_{\left[N, N+N_{2}-1\right]} .
\end{array}
$$

Likewise, (18) can be cast as as: for $l \in \mathbb{N}_{\left[1, n_{c}\right]}$,

$$
f_{(l, 0, \infty)}+\sum_{j=1}^{N+N_{2}-1} f_{(l, 1, j)}+f_{(l, 1, \infty)} \leq 1,
$$

where $f_{(l, 0, \infty)}=\max _{x \in \Omega_{0}}\left(F_{l}+G_{l} K\right) x$, and $f_{(l, 1, \infty)}=$ $\max _{x \in \Omega_{1}^{\infty}}\left(F_{l}+G_{l} K\right) x$. Condition (21) is equivalent to

$$
h_{(l, 0, \infty)}+\sum_{j=1}^{N+N_{2}-1} h_{(l, 1, j)}+h_{(l, 1, \infty)} \leq 1,
$$

for $l \in \mathbb{N}_{\left[1, n_{f}\right]}$, where $h_{(l, 0, \infty)}=\max _{x \in \Omega_{0}} H_{l}^{f} x$, and $h_{(l, 1, \infty)}=$ $\max _{x \in \Omega_{1}^{\infty}} H_{l}^{f} x$. Also, if $\Omega_{0}=\left\{x: H_{l} x \leq 1, l \in \mathbb{N}_{\left[1, n_{f 0}\right]}\right\}$ and $\Omega_{1}=\left\{x: I_{l} x \leq 1, l \in \mathbb{N}_{\left[1, n_{f 1}\right]}\right\}$, then (16) is equivalent to

$$
H_{l} x_{\left(0, N+N_{2}\right)} \leq 1, l \in \mathbb{N}_{\left[1, n_{f 0}\right]},
$$

$$
I_{l} x_{\left(i, 1, N+N_{2}\right)} \leq 1, l \in \mathbb{N}_{\left[1, n_{f 1}\right]}, i \in \mathbb{N}_{[1, q]} .
$$

Remark 10 The minimal invariant set is defined by Minkowski sums of an infinite number of terms, so in practice it cannot be computed exactly. However the approach of [10] can be used to find an outer approximation of $\Omega_{1}^{\infty}$ with arbitrary precision which allows one to invoke (25) and (27) with a conservativeness as small as the precision of the outer approximation.

Through the use of variables $d_{k}$ that act as upper bounds of maxdist $_{X_{f}}\left(X_{k}\right)$, the cost $J(\theta)$ of (20) can be rewritten as

$$
J(\theta)=\sum_{k=0}^{N+N_{2}-1} d_{k},
$$

with the additional constraints, for $k \in \mathbb{N}_{\left[0, N+N_{2}-1\right]}$

$$
\begin{aligned}
& d_{k} \geq H_{l}^{f} x_{(0, k)}+\sum_{j=1}^{k} h_{(l, 1, j)}-1, \quad l \in \mathbb{N}_{\left[1, n_{f}\right]} \\
& d_{k} \geq 0
\end{aligned}
$$

and for $l \in \mathbb{N}_{\left[1, n_{f}\right]}$ and $i \in \mathbb{N}_{[1, q]}$,

$$
\begin{array}{ll}
h_{(l, 1, j)} \geq H_{l}^{f} x_{(i, 1, j)}, & j \in \mathbb{N}_{[1, N]} \\
h_{(l, 1, j)} \geq H_{l}^{f} \Phi^{j-N} x_{(i, 1, N)}, j \in \mathbb{N}_{\left[N+1, N+N_{2}-1\right]}
\end{array}
$$

Clearly the optimal $d_{k}$ will be equal to $\operatorname{maxdist}_{X_{f}}\left(X_{k}\right)$ so the minimization of the cost of (20) and that of (28) are equivalent.

Summarizing, $\mathcal{P}(x)$ is a linear program that minimizes the cost of (28) over $\theta, \mathbf{f}_{\left(l, 1, N+N_{2}-1\right)}=\left\{f_{(l, 1, k)}\right\}_{k \in \mathbb{N}_{\left[1, N+N_{2}-1\right]}}, l \in$ $\mathbb{N}_{\left[1, n_{c}\right]}$, and $\mathbf{h}_{\left(l, 1, N+N_{2}-1\right)}=\left\{h_{(l, 1, k)}\right\}_{k \in \mathbb{N}_{\left[1, N+N_{2}-1\right]}, l \in}$ $\mathbb{N}_{\left[1, n_{f}\right]}$, and $\mathbf{d}_{\left(1, N+N_{2}-1\right)}=\left\{d_{k}\right\}_{k \in \mathbb{N}_{\left[1, N+N_{2}-1\right]}}$ subject to (10a),(10b),(10d),(10e),(24)-(27),(29). $\mathcal{P}(x)$ has $N_{\text {var }}=N\left(n+(1+q)+n_{u}(1+q)+n_{c}+n_{f}+1\right)+N_{2}\left(n_{c}+n_{f}+\right.$ $1)+n-\left(q n_{u}+n_{c}+n_{f}+1\right)$ variables, $N_{e q}=N n(1+q)+n$ equality constraints and $N_{\text {ineq }}=N\left(\left(n_{c}+n_{f}\right)(1+q)+1\right)+$ $N_{2}\left(\left(n_{c}+n_{f}\right)(1+q)+1\right)+n_{c}+n_{f 0}+n_{f 1} q+n_{f}(1-q)$ inequality constraints. Thus, with the striped structure the size of the problem grows linearly with $N$ unlike in [11], where the size of the problem grows quadratically with $N$.

Theorem 11 For system (1) under the control law of (23) the optimal control problem of (22) is recursively feasible and guarantees satisfaction of (2).

Proof: The extension (the "tail") of an optimal solution (indicated by ()$\left.^{*}\right)$ to the next time is

$$
\begin{aligned}
& \tilde{x}_{(0, k)}=x_{(0, k+1)}^{*}(x)+x_{(1, k+1)}(x), k \in \mathbb{N}_{[1, N-1]}, \\
& \tilde{x}_{(0, N)}=\Phi\left(x_{(0, N)}^{*}(x)+x_{(1, N)}\right) \\
& \tilde{u}_{(0, k)}=u_{(0, k+1)}^{*}(x)+u_{(1, k+1)}, \quad k \in \mathbb{N}_{[1, N-2]}, \\
& \tilde{u}_{(0, N-1)}=K\left(x_{(0, N)}^{*}(x)+x_{(1, N)}\right)
\end{aligned}
$$

where $\left(x_{(1, k)}, u_{(1, k)}\right) \in Y_{(1, k)}^{*}(x)$, and

$$
\begin{array}{ll}
\tilde{x}_{(i, 1, k)}=x_{(i, 1, k)}(x), & k \in \mathbb{N}_{[1, N]}, i \in \mathbb{N}_{[1,1]} \\
\tilde{u}_{(i, 1, k)}=u_{(i, 1, k)}(x), \quad k \in \mathbb{N}_{[1, N-1]}, i \in \mathbb{N}_{[1, q]}
\end{array}
$$

The tubes defined by the tail, $\tilde{Y}_{k}$, satisfy $\tilde{Y}_{k} \subseteq Y_{k+1}^{*}(x), k \in$ $\mathbb{N}$. But a feasible current solution satisfies (17)-(18), which guarantees $Y_{k} \subseteq \mathbb{Y}, \quad k \in \mathbb{N}$ so that (17) is guaranteed for $\tilde{Y}_{k}$. Also, because $\tilde{Y}_{(1, k)}=Y_{(1, k)}^{*}(x)$ and $\Omega_{0}$ and $\Omega_{1}$ are fixed, (16b) and (18) are satisfied. The satisfaction of (16a) follows from the fact that $\tilde{x}_{\left(0, N+N_{2}\right)}=\Phi x_{\left(0, N+N_{2}\right)}+\Phi x_{\left(1, N+N_{2}\right)}$ and that $\Omega_{0}$ is invariant for $z^{+}=\Phi z+w$, where $w \in \Phi \Omega_{1}$. Since $\tilde{x}_{(i, 1, k)}=x_{(i, 1, k)}^{*}(x)$, it follows that $\tilde{Y}_{(1, j)}=Y_{(1, j)}^{*}(x)$, and then the tail will satisfy (21) if it is satisfied at the current time. Then if the optimal control problem is feasible, it will remain feasible at all future times. 
Theorem 12 For $x \in \mathcal{X}$, the value function $V^{*}(x)$ satisfies

$$
V^{*}\left(x^{+}\right)-V^{*}(x) \leq-\operatorname{dist}_{X_{f}}(x) .
$$

Proof: From Proposition 3, $X_{k}^{*} \subseteq \operatorname{Proj}_{x}\left(\bar{Y}_{\infty}\right)$ for $k \geq$ $N+N_{2}$, thus from (21) it follows that $X_{k} \subseteq X_{f}$ and $\operatorname{maxdist}_{X_{f}}\left(X_{k}^{*}\right)=0$ for $k \in \mathbb{N}_{\left[N+N_{2}, \infty\right)}$. The definition of the tail of (30)-(31) implies that $\tilde{X}_{k} \subseteq$ $X_{k+1}^{*}(x), k \in \mathbb{N}$ and then the cost at the next sample time satisfies $J(\tilde{\theta})=\sum_{k=0}^{N+N_{2}} \operatorname{maxdist}_{X_{f}}\left(\tilde{X}_{k}\right) \leq$ $\sum_{k=1}^{N+N_{2}} \operatorname{maxdist}_{X_{f}}\left(X_{k}^{*}\right)=V^{*}(x)-\operatorname{dist}_{X_{f}}(x)$. The result follows from maxdist $X_{f}\left(X_{0}^{*}\right)=\operatorname{dist}_{X_{f}}(x)$ and the fact that the optimization ensures $V^{*}\left(x^{+}\right) \leq J(\tilde{\theta})$.

Proposition 13 (i) $\mathcal{X}$ is a polytope that contains the origin in its interior, (ii) $V^{*}(x)=0$ if $x \in X_{f}$ and $V^{*}(x)>0$ if $x \notin X_{f}$.

Proof: (i) Let $\mathcal{C}=\left\{(x, \theta): M_{\theta} \theta+M_{x} x \leq M\right\}$ so that $\mathcal{C}$ is a convex closed polyhedron, and hence $\mathcal{X}$, as the projection of $\mathcal{C}$ onto a suitable subspace, is itself a convex closed polyhedron. If $x \in \mathcal{X}$ then $\exists u_{(0,0)}$ such that $\left(x_{(0,0)}, u_{(0,0)}\right) \in \mathbb{Y}$, with $x_{(0,0)}=x . \mathcal{X}$ is bounded because otherwise there would exist at least one $x$ such that $\lambda x \in \mathcal{X}, \forall \lambda \in \mathbb{R}$, but this contradicts the boundedness of $\mathbb{Y} . \mathcal{X}$ contains the origin in its interior from Assumption 8 as discussed in Remark 9. (ii) If $x \notin X_{f}$ then $\operatorname{dist}_{X_{f}}(x)>0$, and by construction $V^{*}(x)>0$. If $x \in X_{f}$, then $\theta$ given by $\mathbf{x}_{(0, N)}=\left\{x, \ldots, \Phi^{N-1} x\right\}, \mathbf{u}_{(0, N-1)}=$ $\left\{K x, \ldots, K \Phi^{N-1} x\right\}, \mathbf{x}_{(i, 1, N)}=\left\{\tilde{w}_{i} \ldots, \Phi^{N-1} \tilde{w}_{i}\right\}$, and $\mathbf{u}_{(i, 1, N-1)}=\left\{K \tilde{w}_{i} \ldots, K \Phi^{N-1} \tilde{w}_{i}\right\}$ is feasible, and since $X_{f}$ is invariant for $K x, X_{k} \subseteq X_{f}$ for all $k \in \mathbb{N}$.

Corollary 14 For $x_{0} \in \mathcal{X}:$ (i) The value function $V^{*}\left(x_{k}\right) \rightarrow$ 0 as $k \rightarrow \infty$. Furthermore, $V^{*}\left(x_{k}\right) \leq a^{k} V\left(x_{0}\right)$, with $a \in$ $(0,1)$. (ii) The distance function dist $X_{f}(x) \rightarrow 0$ as $k \rightarrow \infty$. Furthermore, dist $X_{X_{f}}\left(x_{k}\right) \leq b^{k}$ dist $_{X_{f}}\left(x_{0}\right)$, with $b \in(0,1)$.

For the proof of this result the interested reader is referred to [11], where analogous results are discussed.

Once the state has reached $\Omega_{K}$, the control law $u=K x$ guarantees constraint satisfaction and ensures that $x$ remains in $\Omega_{K}$. Thus, if instead of solving $\mathcal{P}(x)$, the control input is defined as $u=K x$, then this will steer the state asymptotically to the minimal robustly invariant set $\Omega_{K}^{\infty}$.

The dependence of the domain of attraction $\mathcal{X}$ on the horizons $N$ and $N_{2}$ is now studied. For this, the dependence of $\mathcal{X}$ and $\Omega_{1}$ on $N, N_{2}$ is made explicit by the notation $\mathcal{X}_{N, N_{2}}$ and $\Omega_{1}\left(N, N_{2}\right)$. The desired properties are $\mathcal{X}_{N, N_{2}} \subseteq \mathcal{X}_{N, N_{2}+1}$ and $\mathcal{X}_{N, N_{2}} \subseteq \mathcal{X}_{N+1, N_{2}}$. The following theorem presents an alternative way to construct $\Omega_{0}$ such that the desired nestedness properties are satisfied.

Theorem 15 Let $\Omega_{0}$ be a robustly invariant for system $z^{+}=$ $\Phi z+w$, with $w \in \mathcal{W}$, where $\mathcal{W}$ is any outer approximation of $\operatorname{conv}\left(\Phi^{N_{3}} \mathbb{W} \cup \Phi^{N_{3}+1} \mathbb{W} \cup \Phi^{N_{3}+2} \mathbb{W} \ldots\right), N_{3}>0$. Then the domain of attraction of system (1) under the control law of (23) satisfies the following properties for all $N, N_{2}$ such that $N+N_{2} \geq N_{3}$ :

(i) $\mathcal{X}_{N, N_{2}} \subseteq \mathcal{X}_{N+1, N_{2}-1}$,

(ii) $\mathcal{X}_{N, N_{2}} \subseteq \mathcal{X}_{N, N_{2}+1}$,

(iii) $\mathcal{X}_{N, N_{2}} \subseteq \mathcal{X}_{N+1, N_{2}}$.

Proof: (i) The optimal control problem $\mathcal{P}(x)$ when using $N, N_{2}$ is the same as when using $\tilde{N}=N+1, \tilde{N}_{2}=N_{2}-1$, except that in the former case $u_{(0, N)}$ and $u_{(i, 1, N)}$ are fixed to be $u_{(0, N)}=K x_{(0, N)}$ and $u_{(i, 1, N)}=K x_{(i, 1, N)}$, while in the latter case these are degrees of freedom. In the latter case $u_{(0, N)}$ and $u_{(i, 1, N)}$ can be chosen as $u_{(0, N)}=K x_{(0, N)}$ and $u_{(i, 1, N)}=K x_{(i, 1, N)}$, so the problem for $\tilde{N}$ and $\tilde{N}_{2}$ is feasible whenever it is feasible for $N$ and $N_{2}$. (ii) If $x \in \mathcal{X}_{N, N_{2}}$, then there exists $\theta$ such that (10),(16)-(18),(21) hold. Trivially $\theta$ satisfies (10), (17) for $\tilde{N}=N$ and $\tilde{N}_{2}=N_{2}+1$. Let $\tilde{\Omega}_{1}$ be the updated $\Omega_{1}$ set for $\tilde{N}, \tilde{N}_{2}$ so that $\tilde{\Omega}_{1}=\Phi \Omega_{1}$. Then, since $\Phi^{N_{2}} x_{(i, 1, N)} \subseteq \Omega_{1}$ (from (16)) we obtain that $\Phi^{N_{2}+1} x_{(i, 1, N)} \subseteq \tilde{\Omega}_{1}$ and so (16b) is satisfied. The definition of $\Omega_{0}$ implies that it will not change for different values of $N$ and/or $N_{2}$, and that $\Phi \Omega_{0} \subseteq \Omega_{0}$. This implies that if $x_{\left(0, N+N_{2}\right)} \in \Omega_{0}$ then $x_{\left(0, N+N_{2}+1\right)}=$ $\Phi x_{\left(0, N+N_{2}\right)} \in \Omega_{0}$, and thus (16a) is satisfied. Satisfaction of (18) for $N, N_{2}$ implies that $\bar{Y}_{\infty} \in \mathbb{Y}$. The definition of $\Omega_{1}$ implies that $\Omega_{1}^{\infty}=\tilde{\Omega}_{1}^{\infty} \oplus \Phi^{N_{2}} X_{(1, N)}$, which implies that $\tilde{\bar{Y}}_{\infty}=(I, K) \Omega_{0} \oplus \bigoplus_{j=1}^{N} Y_{(1, j)} \oplus \bigoplus_{j=N}^{N+N_{2}}(I, K) \Phi^{j-N} X_{(1, N)}$ $\oplus(I, K) \tilde{\Omega}_{1}^{\infty}=\bar{Y}_{\infty} \subseteq \mathbb{Y}$, and therefore (18) holds for $\tilde{N}, \tilde{N}_{2}$. Likewise, satisfaction of (21) for $N, N_{2}$ implies that $\operatorname{Proj}_{x}\left(\bar{Y}_{\infty}\right)=\operatorname{Proj}_{x}\left(\tilde{\bar{Y}}_{\infty}\right) \subseteq X_{f}$, thus ensuring satisfaction of (21) for $\tilde{N}, \tilde{N}_{2}$. All this implies that $x \in \mathcal{X}_{N, N_{2}+1}$, which proves the desired result. (iii) This follows directly from combining (i) and (ii).

Remark 16 (i) The construction of $\Omega_{0}$ in (19) is designed so that Assumption 5 is satisfied. However, Theorem 15 establishes a stronger condition on $\Omega_{0}$. A modification of (19) in order to satisfy these new conditions is given by $\Omega_{0}=$ $\alpha \bar{\Omega}_{K} \oplus \tilde{\Omega}_{\mathcal{W}}^{\infty}$, where $\tilde{\Omega}_{\mathcal{W}}^{\infty}$ is an invariant approximation of the minimal invariant set for $z^{+}=\Phi z+w, w \in \mathcal{W}$. (ii) $\mathcal{W}$ may be defined as any outer approximation of (rather than equal to) $\operatorname{conv}\left(\Phi^{N_{3}} \mathbb{W} \cup \Phi^{N_{3}+1} \mathbb{W} \ldots\right)$, in order to reduce the number of vertices or facets needed to define $\mathcal{W}$. (iii) The invariance of $\Omega_{0}$ under $z^{+}=\Phi z+w$ for $w \in \mathcal{W}$ means that it is invariant for $w \in \Phi^{N_{3}} \mathbb{W}, w \in \Phi^{N_{3}+1} \mathbb{W}$, etc. Thus the recursive feasibility established in Theorem 11 still holds, but only if $N+N_{2} \geq N_{3}$ (due to the definition of $\Omega_{1}$ ). As a result, $N_{3}$ should be chosen small enough as dictated by practical values of $N, N_{2}$, but big enough to maintain $\Omega_{0}$ small.

\subsection{Nominal cost and input-to-state stability}

This section presents a summary of a variation of the strategy of Section 4.1 (which was presented in [8]). This strategy uses a nominal type of cost and does not use the terminal condition of (21). This variation therefore has more freedom, thereby allowing for greater domains of attraction and has a reduced number of variables and constraints, but does not guarantee convergence to the minimal robust invariant set, $\Omega_{K}^{\infty}$.

The objective function is defined as the nominal cost:

$$
J(\theta)=\sum_{k=0}^{N-1} x_{(0, k)}^{T} Q x_{(0, k)}+u_{(0, k)}^{T} R u_{(0, k)}+x_{(0, N)}^{T} P x_{(0, N)}
$$

where $Q, R, P \succ 0$, and satisfy the Lyapunov inequality $\Phi^{T} P \Phi-P \leq-\left(Q+K^{T} R K\right)$.

We redefine $\Theta(x)$ as $\Theta(x)=\{\theta$ : s.t. (10a),(10b),(10d), (10e),(16),(17),(18) hold $\}$. Then $\mathcal{X}$ and $\mathcal{P}(x)$ are modified according to the new $\Theta(x)$ and $J(\theta)$. The control law is given by (23) but according to the modified $\mathcal{P}(x)$, and the input is then set to $u:=\kappa^{0}(x)$.

Remark 17 The results of Theorem 11 (recursive feasibility) and of Theorem 15 (nestedness of $\mathcal{X}_{N, N_{2}}$ ) are also valid for this variation of the strategy since removing condition (21) does not change the analysis. 
On the other hand, the stability properties of Corollary 14 do not hold without (21) and with the cost of (33). Instead we consider input-to-state stability (ISS) [5]. A summary of the key points is presented next; the interested reader is referred to [2] and [8], where analogous ISS results are discussed.

Assume time-invariant nonlinear dynamics $x^{+}=f(x, w)$ where $x \in \mathbb{R}^{n_{x}}$ is the state, $w$ is a disturbance that lies in a compact set $\mathbb{W} \subset \mathbb{R}^{n_{w}}$, and $f(\cdot, \cdot)$ is continuous such that $f(0,0)=0$.

Definition 18 For the system $x^{+}=f(x, w)$, the origin is ISS in $\mathcal{E} \in \mathbb{R}^{n_{x}}$, which contains the origin in its interior, if there exist a $\mathcal{K} \mathcal{L}$-function $\beta(\cdot, \cdot)$ and a $\mathcal{K}$-function $\gamma(\cdot)$ such that, for all $x_{0} \in \mathcal{E}$ and all admissible disturbances $w \in \mathbb{W}$, the solution of $x^{+}=f(x, w)$ satisfies $\forall k \in \mathbb{N}$

$$
\left\|x_{k}\right\| \leq \beta\left(\left\|x_{0}\right\|, k\right)+\gamma\left(\sup \left\{\left\|w_{t}\right\|: t \in \mathbb{N}_{[0, k-1]}\right\}\right) \text {. }
$$

Remark 19 Following a similar analysis to that of [2] and [8], it can be proved that the origin is ISS for system (1) under the control law $u:=\kappa^{0}(x)$, with domain of attraction $\mathcal{X}$. ISS imples that: (i) the origin is asymptotically stable for $x^{+}=f(x, 0)$ with domain of attraction $\mathcal{E}$; (ii) all state trajectories are bounded for bounded $w_{k}$; and (iii) as $k \rightarrow \infty$ all trajectories go to the origin if $w_{k} \rightarrow 0$.

\section{Illustrative example}

The benefits of the proposed strategy are illustrated by a simulation example. Consider a system defined by $A=\left[a_{1}^{T}, a_{2}^{T}\right]^{T}$ with $a_{1}=[0.787,-0.933]$, and $a_{2}=[1.015,1.033]$, and $B=[0.331,-1.006]^{T}$. Let $\mathbb{W}$ be the square with corners $[ \pm 1, \pm 1]^{T}$ and $\mathbb{Y}=\{(x, u): \pm[-0.044,0.092] x \leq 1$, $\pm[0.009,0.093] x \leq 1, u \leq 1,-u \leq 1\}$. $\Omega_{0}$ is constructed according to Remark 16 with $N_{3}=6, \alpha=0.01$ and to ensure satisfaction of Assumption 8 we set $N_{2}=5$. Table 1 shows the areas of the domains of attraction $\left(A_{N}\right)$, the number of online optimization variables (Vars), equality (Eqs) and inequality constraints (Ineqs) when using SPTMPC1, SPTMPC2 (the strategies of Sections 4.1 and 4.2) and PTMPC [11] (under columns M1, M2 and M3 respectively). The rows of Table 5 present the results for different prediction horizons $N$.

When $N \geq 4$, SPTMPC1,2 yield larger domains of attraction than PTMPC for equal $N$. This is mainly due to the fact that disturbance compensation in SPTMPC enters into Mode 2 , thereby relaxing the terminal constraints. PTMPC has a full triangular structure, which is more general and therefore, depending on the example, can outperform SPTMPC. However, the price of the full triangular structure is that it implies a computational load that grows quadratically with $N$, whereas the online computation of SPTMPC grow only linearly with $N$. Thus with SPTMPC one can use longer horizons, thereby enlarging the size of the domain of attraction, at a computational cost which is still less than that required by PTMPC. For example, while for $N<4$ SPTMPC1,2 have more variables and inequality constraints, for $N \geq 4$ SPTMPC1,2 lead to larger domains of attraction while using fewer variables and constraints. Furthermore, due to the quadratic increase (in number of variables and constraints) in PTMPC versus the linear increase in SPTMPC1,2, larger values of $N$ can be used in SPTMPC1,2, thus obtaining even larger domains of attractions and still using fewer variables and constraints (e.g. see the case of SPTMPC1,2 with $N=10$, and PTMPC with $N=5$ ).

Finally, SPTMPC2 does not constrain $x_{N+N_{2}}, x_{N+N_{2}+1}, \ldots$ to lie inside $X_{f}$ and hence it achieves larger domains of attraction with fewer variables than SPTMPC1. However the latter guarantees convergence to $\Omega_{K}$ where $u=K x$ can be applied thus providing convergence to $\Omega_{K}^{\infty}$.
Table 1: Domain of Attraction and Computational Aspects

\begin{tabular}{|c|c|c|c|c|c|c|c|c|c|c|c|c|}
\hline $\mathrm{N}$ & $\begin{array}{l}A_{N} \\
\mathrm{M} 1\end{array}$ & M2 & M3 & $\begin{array}{l}\text { Vars } \\
\text { M1 }\end{array}$ & M2 & M3 & $\begin{array}{c}\text { Ineqs } \\
\text { M1 }\end{array}$ & M2 & M3 & $\begin{array}{l}\text { Eqs } \\
\text { M1 }\end{array}$ & M2 & M3 \\
\hline 1 & 2.38 & 2.38 & 2.38 & 93 & 67 & 24 & 296 & 186 & 61 & 2 & 2 & 10 \\
\hline 2 & .01 & 2.87 & 3.01 & 120 & 89 & 71 & 347 & 216 & 158 & 12 & 12 & 28 \\
\hline 3 & 57 & 3.57 & 3.66 & 147 & 111 & 144 & 398 & 246 & 303 & 22 & 22 & 54 \\
\hline 4 & 4.44 & 4.53 & 4.19 & 174 & 133 & 243 & 449 & 276 & 496 & 32 & 32 & 88 \\
\hline 5 & 5.02 & 5.14 & 4.59 & 201 & 155 & 368 & 500 & 306 & 737 & 42 & 42 & 130 \\
\hline 6 & 5.25 & 5.39 & 4.88 & 228 & 177 & 519 & 551 & 336 & 1026 & 52 & 52 & 180 \\
\hline 7 & 5.44 & 5.83 & 5.13 & 255 & 199 & 696 & 602 & 366 & 1363 & 62 & 62 & 238 \\
\hline 8 & 5.57 & 5.87 & 5.34 & 282 & 221 & 899 & 653 & 396 & 1748 & 72 & 72 & 304 \\
\hline 9 & 5.66 & 5.95 & 5.48 & 309 & 243 & 1128 & 704 & 426 & 2181 & 82 & 82 & 378 \\
\hline 10 & 5.72 & 5.95 & 5.59 & 336 & 265 & 1383 & 755 & 456 & 2662 & 92 & 92 & 460 \\
\hline
\end{tabular}

\section{Conclusions}

Deploying a predicted control law where the degrees of freedom directly affect the predicted inputs over an infinite horizon, a RMPC strategy is proposed with numbers of variables and constraints that grow only linearly with the prediction horizon, and domains of attraction that are potentially larger than those of PTMPC. Two variations of the strategy are discussed, one allowing greater improvements in the domain of attraction and input-to-state stability, and the other providing convergence to a terminal invariant set for $u=K x$.

\section{References}

[1] D. Bertsekas. Dynamic Programming and Optimal Control. Athena Scientific, 1995.

[2] P. Goulart, E. Kerrigan, and J. Maciejowski. Optimization over state feedback policies for robust control with constraints. Automatica, 42(4):523 - 533, 2006.

[3] B. Kouvaritakis, M. Cannon, and D. Muñoz-Carpintero. Efficient prediction strategies for disturbance compensation in stochastic MPC. Int. J. of Systems Science, 44(7):1344-1353, 2013.

[4] W. Langson, I. Chryssochoos, S.V. Raković, and D.Q. Mayne. Robust model predictive control using tubes. Automatica, 40(1):125 - 133, 2004.

[5] M. Lazar, D. Muñoz de la Peña, W.P.M.H. Heemels, and T. Alamo. On input-to-state stability of minmax nonlinear model predictive control. Systems \& Control Letters, 57(1):39 - 48, 2008.

[6] Y. I. Lee and B. Kouvaritakis. Constrained receding horizon predictive control for systems with disturbances. International Journal of Control, 72(11):1027-1032, 1999.

[7] J. Lofberg. Minimax approaches to robust model predictive control. $\mathrm{PhD}$ thesis, Department of Electrical Engineering, Linkoping University, Linkoping, Sweden, 2003.

[8] D. Muñoz-Carpintero, B. Kouvaritakis, and M. Cannon. Striped parameterized tube model predicted control. In IFAC World Congress, 2014.

[9] S.V. Raković and M. Baric. Parameterized robust control invariant sets for linear systems: Theoretical advances and computational remarks. Automatic Control, IEEE Transactions on, 55(7):1599-1614, 2010.

[10] S.V. Rakovic, E. Kerrigan, K. Kouramas, and D. Mayne. Invariant approximations of the minimal robust positively invariant set. IEEE Transactions on Automatic Control, 2005.

[11] S.V. Raković, B. Kouvaritakis, M. Cannon, C. Panos, and R. Findeisen. Parameterized tube model predictive control. IEEE Transactions on Automatic Control, 57(11):2746 - 2761, 2012.

[12] P. Scokaert and D. Mayne. Min-max feedback model predictive control for constrained linear systems. Automatic Control, IEEE Transactions on, 43(8):1136 -1142, August 1998. 